Peer Reviewed Paper openaccess

\title{
A novel dual-path high-throughput acousto- optic tunable filter imaging spectropolarimeter
}

\author{
Ramy Abdlatya,* and Qiyin Fang ${ }^{b}$ \\ aDepartment of Biomedical Engineering, Military Technical College, Cairo, Egypt \\ bEngineering Physics, McMaster University, Hamilton, Ontario, Canada \\ Contacts \\ R. Abdlaty: ramy.elghwas@mtc.edu.eg; \\ https://orcid.org/0000-0002-8933-1724 \\ Q. Fang: qiyin.fang@mcmaster.ca; \\ https://orcid.org/0000-0003-2786-9884
}

\begin{abstract}
It is highly demanding to identify healthy and non-healthy species in a heterogeneous environment such as human tissues. In such a case, one identifier, such as a spectral fingerprint, might be inadequate. Therefore, additional identification is required, for instance, a polarisation measurement. In view of that, the development of a spectropolarimeter that captures two cross-polarised arrays of spectral images is a key requirement. To meet this requirement, an imager optical setup has been designed to provide spatial, spectral and polarisation preference information for species that exist in a heterogeneous environment, such as in medical tissue samples. The spectral and polarisation information is obtained employing an acousto-optic tunable filter and a polarising beam splitter, respectively. The optical imager is designed to operate in the visible-near infrared range $(450-850 \mathrm{~nm})$ with a spectral resolution of $3 \mathrm{~nm}$. The spectropolarimeter design along with optical characterisation results are reported.
\end{abstract}

Keywords: hyperspectral imaging, polarised imaging, spectral footprint, AOTF, optical design

\section{Introduction}

Human tissue samples are in many cases confusing, heterogeneous environments for identifying disease. ${ }^{1}$ Hyperspectral imaging (HSI) is a contactless inspection technique that has been employed for identification purposes in medical tissues such as skin inflammation levels, and in vitro atherosclerosis levels in human veins. ${ }^{2,3}$ Nonetheless, solely the spectral footprint in some medical situations is insufficient. ${ }^{4}$ Consequently, a second identifying mark for tissue is recommended to assist physicians in their critical role of diagnosis or treatment.

$\mathrm{HSI}$ is a promising technology for contactless inspection and precise remote sensing for various objects (tissues, fluids etc.). The potential of HSI is built upon the abundance of identifying information for the object of interest. HSI information holds the form of a threedimensional matrix. This matrix has two spatial dimensions for the object's region of interest and one dimension for a spectral record for each spatial location. 5,6 HSI has displayed a profound impact in a diversity of research fields including archaeology, wastewater monitoring, food industry, criminology, forensics, medicine and planetary remote sensing. ${ }^{7-14}$ Nevertheless, the valuable information of HSI might disguise evidence of the presence of some species in a heterogeneous environment. ${ }^{11,15,16}$

\section{Correspondence}

Ramy Abdlaty (ramy.elghwas@mtc.edu.eg)

Received: 30 October 2020

Revised: 18 December 2020

Accepted: 21 December 2020

Publication: 27 December 2020

doi: 10.1255/jsi.2020.a20

ISSN: 2040-4565

\begin{abstract}
Citation
R. Abdlaty and Q. Fang, "A novel dual-path high-throughput acousto-optic tunable filter imaging spectropolarimeter", J. Spectral Imaging 9, a20 (2020). https://doi.org/10.1255/jsi.2020.a20

(c) 2020 The Authors

This licence permits you to use, share, copy and redistribute the paper in any medium or any format provided that a full citation to the original paper in this journal is given, the use is not for commercial purposes and the paper is not changed in any way.
\end{abstract}


For such cases, a piece of auxiliary information might improve identification. One approach, for identification of paradoxes, is the polarisation preference for an object (the polarisation preference of an object means that this object gives maximum display when the incident light is polarised and its polarisation direction matches its preference). ${ }^{17-24}$ Accordingly, the acquisition of a group of distinguishing features such as the spectral, the spatial and the polarisation preference information for an object has a high potential.

In addition to the acquisition of the object's spatial and spectral features, various optical designs were earlier proposed for HSI systems. ${ }^{25-28}$ These designs include optical filter-based-imaging spectrometers ${ }^{29-31}$ and imaging spectrographs via prisms or gratings. ${ }^{32,33}$ Among the variety of designs, optical tunable filter (OTF)-based $\mathrm{HSI}$ is an emerging research direction. ${ }^{34-37}$ The OTF is a controlled window that only permits a user-selected spectral band to pass. The controlling phenomena allow sorting of optical filters into different types. ${ }^{29}$ Two types of OTF are more dominant in HSI designs: liquid crystal tunable filter (LCTF) ${ }^{37,38-40}$ and acousto-optic tunable filter (AOTF). ${ }^{18,21-23}$ These dominant types of OTF have been compared in a few studies. ${ }^{34,41,42}$ The AOTF enables: (1) rapid random access to spectral bands, (2) relatively long spectral range of operation, (3) robust performance in harsh environments ${ }^{17,43}$ and (4) wide aperture within compact size-tunable filters. ${ }^{21}$ For all of these reasons, we used AOTF in this study.

AOTF-based-imaging spectrometers have two conventional configurations. One configuration employs two image detectors in order to capture the diffracted orthogonally polarised spectral images simultaneously.4.45 This configuration is limited by a small field of view (FOV) as it captures two spectral images at once. The other AOTFspectrometer configuration uses a single detector and substitutes the second with an external variable retarder in order to alter the polarisation of the input light. 17,21,46 This configuration overcomes the limited FOV, nevertheless, it lacks the facility of capturing two orthogonally polarised images. For a rapidly changing biological sample occupying a wide FOV, a novel configuration needs to be proposed.

In this paper, we propose a novel design for a dual-path AOTF-HSI optical configuration. The novel configuration captures two orthogonally polarised datacubes simultaneously for a FOV of $13 \mathrm{~cm}^{2}$ at a distance of $70 \mathrm{~cm}$. The proposed AOTF-HSI instrument runs in the visible-near infrared (VNIR) spectral zone, $450-850 \mathrm{~nm}$, limited by a spectral resolution range of $1.5-5 \mathrm{~nm}$. In general, the image acquisition time for a whole datacube is $90 \mathrm{~s}$, taking into consideration that $1 \mathrm{~s}$ is required for each frame. The spatial resolution for the developed AOTF-HSI instrument is measured using a USAF-1951 object. The smallest target clearly resolved by the AOTF-HSI is group 1/element-1 which is equivalent to $2 \mathrm{LP} \mathrm{mm}^{-1}$. The optimal location of the target to be in focus was determined to be within $668 \mathrm{~mm}$ up to $702 \mathrm{~mm}$, therefore, the effective depth of focus is equal to $34 \mathrm{~mm}$. To characterise the dual-path AOTF-HSI instrument, three targets were imaged: (1) a ceramic painted cup, in order to evaluate the polarisation preference, (2) a spectral calibration target, for calibration purposes and (3) a passport colour checker to evaluate the imager spectral performance.

\section{Materials and methods}

Two halogen sources emitting linearly polarised light were used to evenly illuminate the object's region of interest (ROI) in order to be imaged by the dual-path AOTF-HSI instrument. The AOTF-HSI system, shown in Figure 1, starts with a zoom lens in order to collect the back-scattered light. This lens forms an image of the ROI at the lens' focal plane. At this plane, a square aperture is installed to allow only the image of the ROI to be clear of any optical noise. A second lens is installed at a focal length far from the ROI image in the optical setup. This lens is the primary one in a two-lens optical relay used in building the dual-path AOTF-HSI instrument. The optical relay transfers the ROI image to the image sensor. The relay lenses are achromatic; in order to reduce the chromatic aberration effect. In the region between the two relay lenses, a polarising beam splitter (PBS) is installed. The PBS internally divides the input optical beam into two orthogonally polarised collimated rays. One is transmitted and one is perpendicularly reflected. The reflected ray meets a mirror installed on the right-hand side of the PBS in order to direct the ray toward the input aperture of the AOTF crystal. On the way toward the AOTF crystal, the reflected PBS ray passes through a half-wave plate in order to modify its polarisation. After this modification, the direction of polarisation for both rays out of the PBS are adjusted to match the AOTF crystal optimal input polarisation.

The novel HSI optical setup, in Figure 1, incorporates a non-collinear AOTF device (manufactured by Gooch \& Housego TF625-350-2-11-BR1A) optimised for imaging applications. The incorporated AOTF device contains a crystal that is optimally sensitive to vertically polarised incident light. The filter crystal has an angular acceptance 


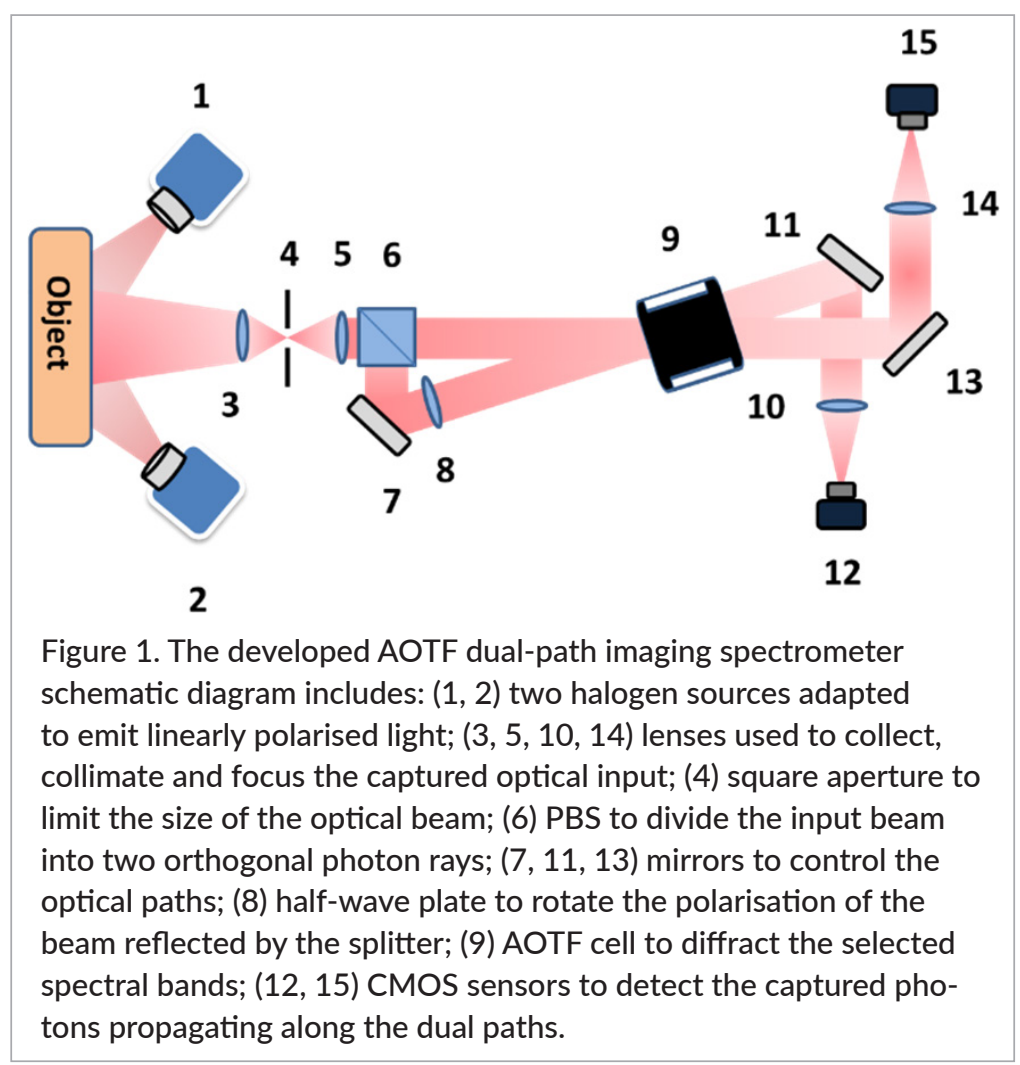

limited to $\pm 2^{\circ}$ for input optical rays. For tuning the optical output of the filter crystal, a radio frequency (RF) signal ranging from $40 \mathrm{MHz}$ up to $150 \mathrm{MHz}$ is applied. The RF tuning signal is generated by the RF signal synthesiser (manufactured by Gooch \& Housego MSD040-150$0.2 \mathrm{ADM}-\mathrm{A} 5 \mathrm{H}-8 \times 1$ ). The generated RF signal reaches the AOTF crystal through a piezoelectric transducer. The transducer, in response to the RF signal, generates a train of acoustic waves that propagate through the AOTF crystal. The acoustic waves transform the AOTF crystal into a transient diffraction grating for the input light beam. The non-collinear AOTF diffraction forces a user-selected optical narrowband to be deviated out of the input broadband beam. To tune the deviated band along the AOTF operating range, the user sequentially changes the RF signal from one end to the other.

The non-collinear AOTF is accurately aligned to allow the PBS output rays to propagate through the crystal within input angular acceptance. The propagating rays through the AOTF crystal are subject to diffraction that causes a narrowband from each ray to be separated. The separated narrowband from each ray deviates, by $4.5^{\circ}$, at the exit aperture of the AOTF device. Before the end of the imager optical path, two mirrors were installed, one for each separated narrowband, in order to direct them toward the second lens of an optical relay. Each optical relay forms the ROI image with a unity magnification limited by the diffracted narrowband at two CMOS image sensors. The sensors' active region is divided into $2048 \times 2048$ pixels in a camera housing (Ximea MQ042rg-CM enhanced-IR). This camera has several advantages, including compact size, high quantum efficiency, large sensor format, low power consumption and low dark noise. In the final analysis, the developed imager setup will capture two simultaneous orthogonally polarised images for an object.

In terms of polarisation, Figure 2 demonstrates the optical beam path in the developed spectropolarimeter. The path starts with a linearly polarised light beam emitted from two halogen sources in order to illuminate the object of interest. The back-scattered light, which is randomly polarised, is captured and directed toward the PBS. The PBS divides the randomly polarised input light into vertically (transmitted) and horizontally (reflected) polarised light rays. Next to the PBS, the installed AOTF cell is optimised for high throughput when a vertically polarised optical beam is input. The PBS transmitted ray matches the installed AOTF cell high-throughput condition, but the reflected ray does not. Therefore, a halfwave plate is installed in order to transform the PBS reflected ray into a vertical polarised one. From this point on, the PBS output rays become vertically polarised. 
The AOTF-HSI imager simultaneously captures two orthogonally polarised datacubes for the object's ROI. It was of interest to evaluate the consistency of the imager's optical paths; for this purpose, the diffracted rays along each path were studied in terms of the tuning RF signal and the narrowband linewidth. In theory, the AOTF laws for the tuning RF signal for a certain spectral band are shown in Equation 1, and the spectral linewidth for the diffracted narrowband beam is shown in Equation 2. The terms used in Equations 1 and 2 are as follows: $\lambda$ is the wavelength, $\nu$ is the acoustic speed, $f$ is the frequency of the acoustic wave, $\Delta n$ is the difference between the indices of refraction for the incident and diffracted radiations, $\theta_{i}$ is the angle of the optical incident ray, $\Delta \lambda$ is the full-width half maximum of the AOTF-diffracted band, $b$ is the spectral dispersive constant of the AOTF cell and $L$ is the length of interaction between the acoustic wave and optical radiation.

$$
\lambda=\frac{v \Delta n}{f} \sqrt{\sin ^{4} \theta_{i}+\sin ^{2} 2 \theta_{i}}
$$

$$
\Delta \lambda=1.8 \pi \lambda^{2} / \mathrm{bL} \sin ^{2} \theta_{i}
$$

Tuning the AOTF cell is achieved by the emitted RF signal generated by the AOTF driver. Each RF signal enables the AOTF cell to diffract one narrowband in two directions. One direction is tagged the positive diffraction order and the other is tagged the negative diffraction order. The diffracted narrowband, for each order per each RF signal, is measured by a high-resolution spectrophotometer (Newport-OSM-400, Irvine, CA, USA). The measured central wavelength for each diffracted narrowband, in the two first diffracted orders of the AOTF cell, is plotted against the generated RF frequency of the AOTF synthesiser in Figure 3(a). The figure illustrates a spectral shift between the central wavelengths of the positive and negative diffracted orders. The spectral shift ranges from $2 \mathrm{~nm}$ to $16 \mathrm{~nm}$. In Figure 3(b), the spectral linewidth for both AOTF-diffracted orders against the spectral range of AOTF operation is displayed. Figure 3(b) shows that the linewidth is not exactly the same for the positive

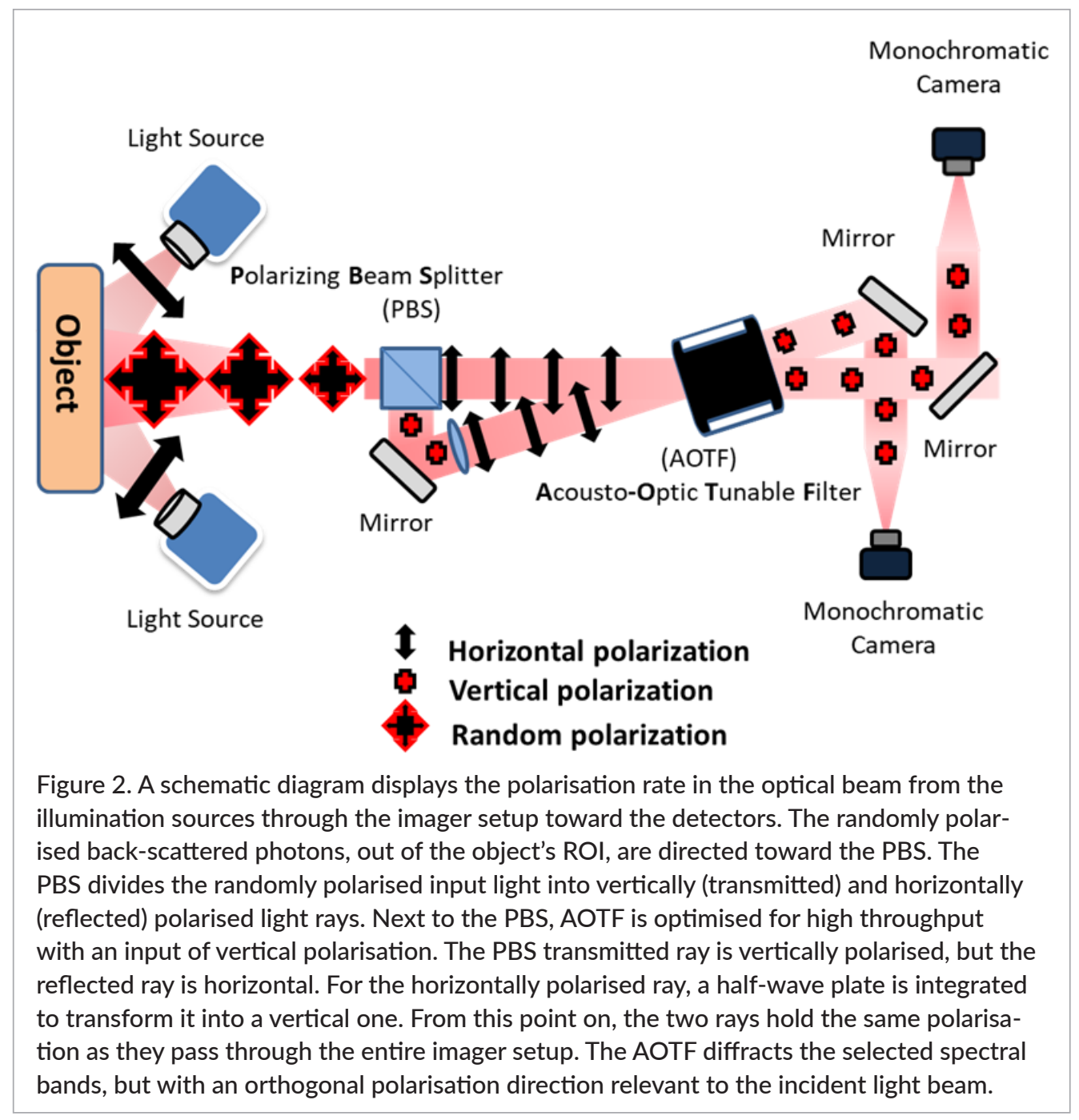




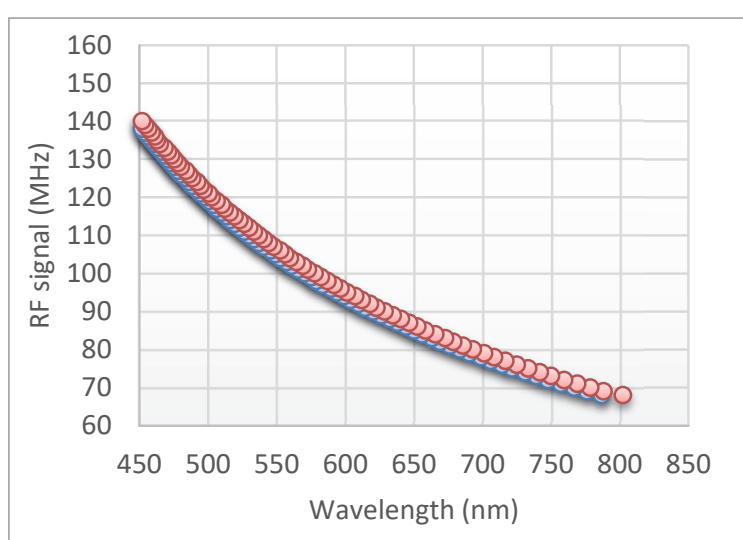

O Negative order O Positive order

(a)

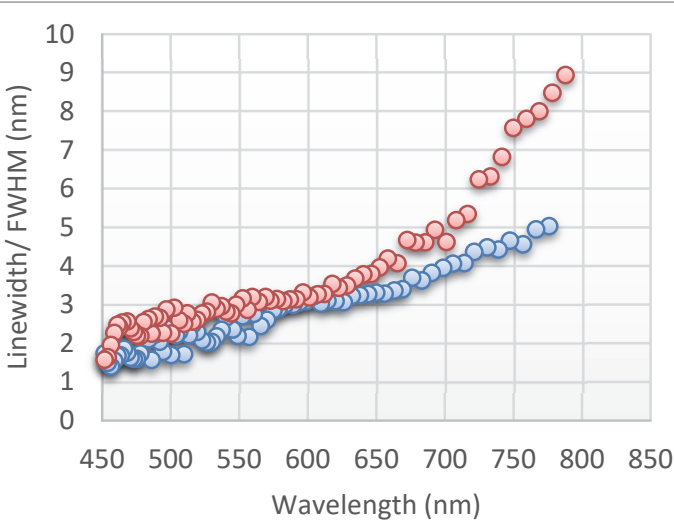

O Negative order O Positive order

(b)

Figure 3. (a) The AOTF-HSI imaging spectrometer tuning relationship for both negative (blue circles) and positive (red circles) first diffracted orders. (b) The AOTF-HSI imaging spectrometer linewidth (spectral resolution) for both negative (blue circles) and positive (red circles) first diffracted orders.

and negative orders. Accordingly, the spectral imaging using both diffracted orders of an optimised AOTF cell is meaningless. Figure 3(b) shows also that the negative order has a higher spectral resolution (lower linewidth) compared to the positive order. Therefore, building an imaging spectrometer employing the optimised AOTF crystal enables imaging of a large spatial FOV within a narrow spectral window.

As there are two channel paths in the breadboard imager setup, a comparison between the linewidth for each path is of interest for quality purposes. Figure 4 shows that the maximum difference detected between the two optical paths does not exceed $0.6 \mathrm{~nm}$. The detected spectral

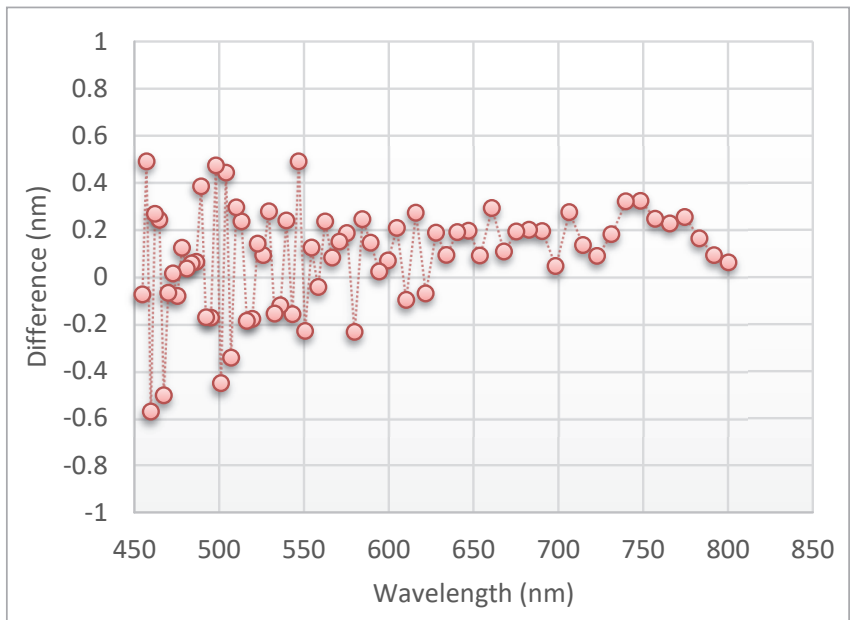

Figure 4. The AOTF imaging spectrometer dual paths linewidth difference plotted along the entire range of operation. difference between the two optical paths is less than the imaging spectrometer's finest spectral resolution.

\section{Experimental imaging}

For polarised imaging, a white ceramic painted object, which is shown in Figure 5(a), is imaged. The selected object is characterised by 1) a cylindrical shape, to show the ability of imaging non-flat surfaces, 2) a painted appearance with several colours to produce images at subsequent spectral bands and 3) a glossy surface to highlight the hurdle of the specular reflection in spectral imaging.

In addition, two diffuse reflectance targets are imaged to determine the value of the impact of the polarisation preference on the spectral signature. The first target is a wavelength calibration target, erbium oxide (LabsphereWCS-EO-010-2169) as shown in Figure 5(b). This target is also used to spectrally assess the two optical paths of the developed imager. ${ }^{47}$ The second target is an X-Rite Color Checker Passport (CCP), as shown in Figure 5(c). The CCP target is commonly used for photography correction and spectral camera characterisation. ${ }^{48}$ The target's datacubes are corrected for the detector's dark noise. The noise-free datacubes are normalised to a standard white target (Labsphere-SRS-99) as shown in Equation 3.

$$
R_{n}=\frac{I_{T}-D}{I_{s 99}-D}
$$




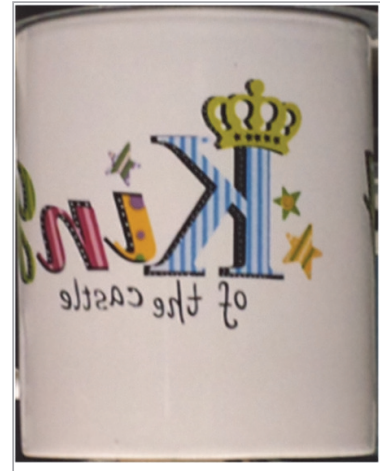

(a)

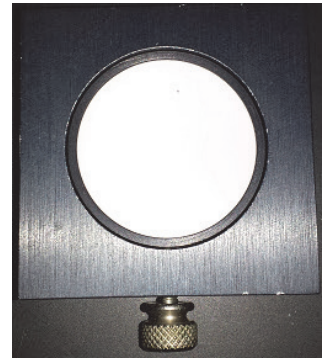

(b)

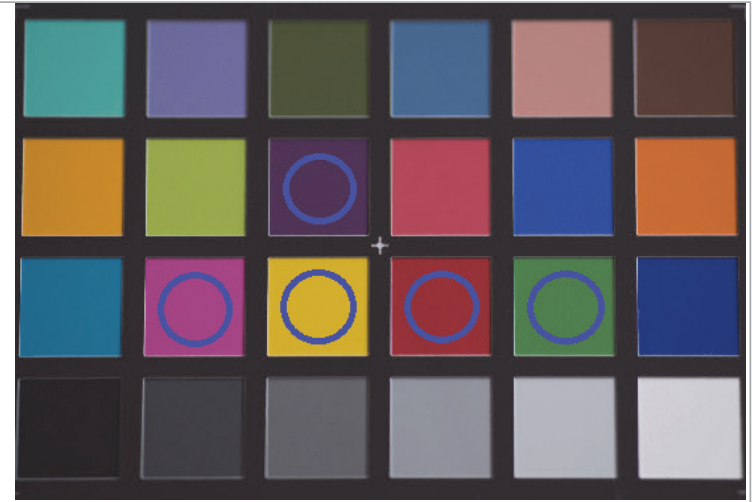

(c)

Figure 5. (a) A white ceramic painted object is imaged to assess the benefit of combining the polarisation against the spectra as two identification parameters in the developed hyperspectral imager. (b) One-inch wavelength calibration diffuse reflectance target, erbium oxide (Labsphere-WCS-EO-010-2169) and (c) $X$-Rite CCP were used to examine the spectral variation of the mean pixels' reflectance intensities of the captured two orthogonal polarisations.

$R_{n}$ is the computed reflectance of the object's FOV, I $I_{599}$ is the reflected intensity of the white target, $I_{T}$ is the reflected intensity of the object's FOV and D is the detector's dark intensity. The complete reflectance for each pixel in the FOV is a sequence of data extracted from the entire spectral bands using Image-J freeware. ${ }^{49}$

\section{Results}

\section{Polarised imaging results}

To illustrate the benefits of cross-polarised imaging, we compared the captured images for the glossy object at two orthogonal (vertical and horizontal) polarisations. A set of datacubes was captured by the developed spectropolarimeter for the ceramic object. The captured dataset contains three cubes, one for each polarisation, and one for both polarisations combined. The same
FOV was imaged by a digital colour camera in the same position as the spectropolarimeter. A group of images, one colour and one spectral from each datacube for the ceramic object, is displayed in Figure 6. The displayed spectral images are selected to be the ones captured in the narrowband that was centred at a wavelength of $602.5 \mathrm{~nm}$. This specific narrowband was chosen for display because it has the highest signal-to-noise ratio within the datacube.

As seen in Figure 6(a), some specular reflection is apparent from the ceramic object colour image. The shiny specular reflection obstructs the vision of the horizontally flipped K-shape and part of the painted crown. The spectral images of the object captured by the vertically polarised light path or by both polarisations combined are obstructed by the same specular reflection. In contrast, the image captured using the horizontally polarised light path clearly shows the flipped K-shape. For verification

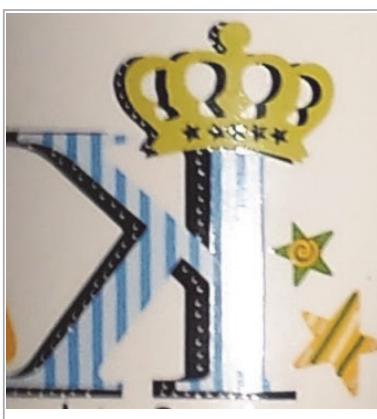

(a)

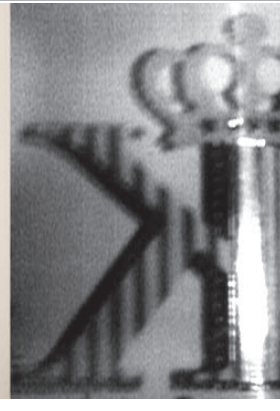

(b)

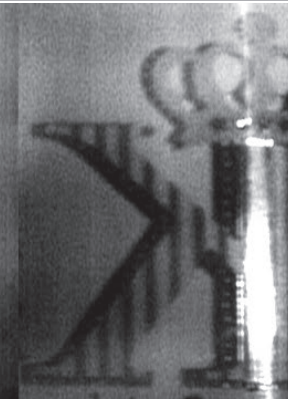

(c)

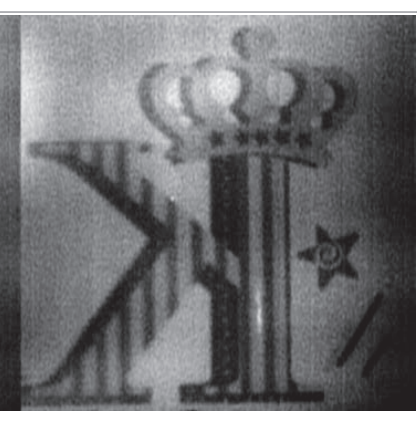

(d)

Figure 6. (a) Ceramic object captured with digital colour camera; (b) the same FOV captured with the breadboard HSI camera at $602.5 \mathrm{~nm}$ central wavelength with two orthogonal polarisations combined; (c) the same FOV but only using vertical polarisation; and (d) the same FOV but only using horizontal polarisation. 
purposes, we repeated exactly the same imaging procedure, but instead, we rotated the direction of the illumination polarisation by $90^{\circ}$. As a result, the vertically and the horizontally polarised images were replaced (images are not shown) and this time the vertically polarised light path clearly shows the flipped K-shape. Accordingly, capturing images using a single polarisation, Figure 6(c), or combining two orthogonal polarisations, Figure $6(b)$, may not be sufficient to retrieve details obstructed by the specular reflection. However, the presence of the two cross-polarised images separated is enough to reveal some lost details as shown in Figure 6(d).

\section{Spectral imaging results}

Figure 7 displays the computed reflectance of a wavelength calibration reflectance (erbium oxide) target. The red circles represent the vertically polarised backscattered light and the green ones represent the horizontally polarised light. The purpose of imaging the calibration target is to substantiate the spectral performance of the spectropolarimeter. For this purpose, an erbium oxide target was imaged since it has two absorption peaks in the VNIR spectral region at the ranges of $521.4-523.4 \mathrm{~nm}$ and 653.8-656.4 nm, respectively. The breadboard spectropolarimeter succeeded in detecting these erbium oxide absorption peaks in the spectral range specified by the manufacturer, as shown in Figure 7.

Figure 5(c) shows a CCP target. In the target, five different colours, marked by blue circles, are selected. The selected five colours were randomly selected out of 24 colours in order to investigate its polarisation preference. The computed reflectance for each colour is displayed in Figure 8(a) for the horizontally polarised light path and in Figure 8(b) for the vertically polarised one. The reflectance of the selected five colours showed a similar spectral profile for both polarisations. However, the intensities of the computed reflectance are not the same. The intensity variation is attributed to the polarisation preference of the CCP target for one polarisation over the other. The displayed curves are the linear leastsquare fitting of the data points at a confidence interval of $95 \%$.

\section{Discussion}

Driven by the benefit of the polarisation and the spectral identities of different objects, a mobile VNIR spectropolarimeter has been developed. The spectropolarimeter is built using an AOTF device for optical dispersion and polarising beam splitting for cross-polarised light designation. The significance of cross-polarised light imaging was emphasised via imaging a man-made object. In fact, polarised light imaging overcomes the specular reflection that obstruct essential details.

A wavelength calibration target was used to scrutinise the developed spectropolarimeter at both polarisations. The calibration target displayed no preferential polarisation, as it maintained the same spectral behaviour regardless of the polarisation of the captured light. In the CCP, variable intensity for each colour was detected. This variability verified the presence of a polarisation preference

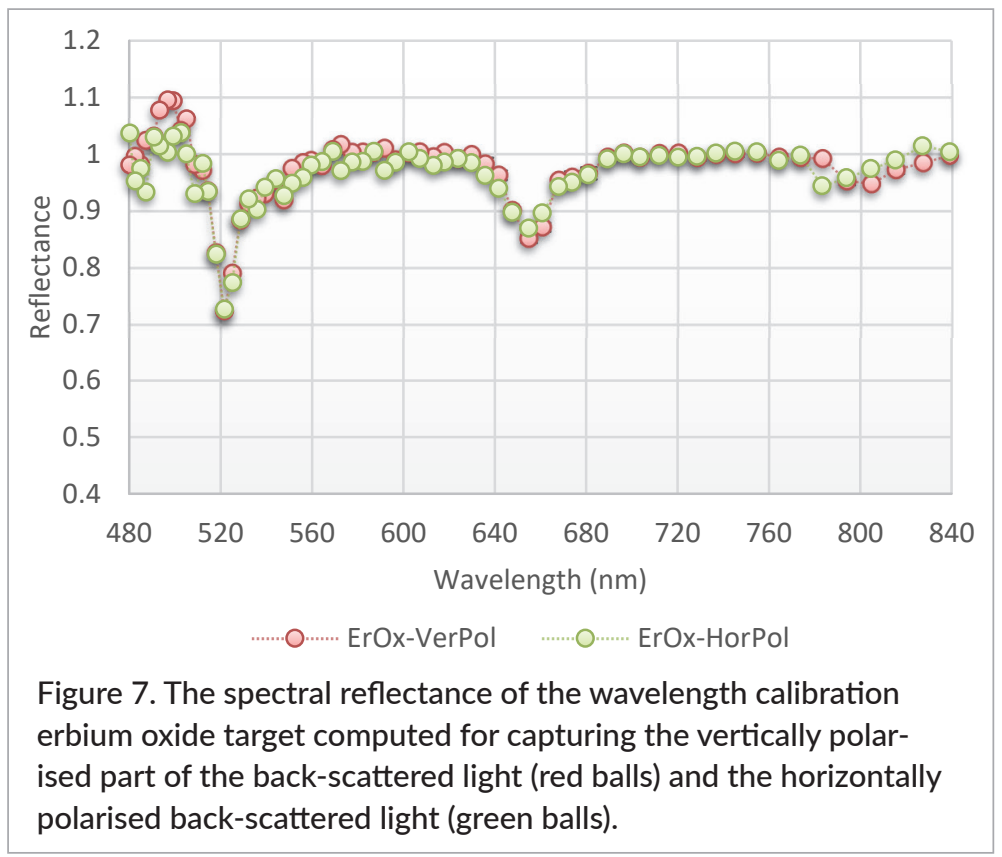




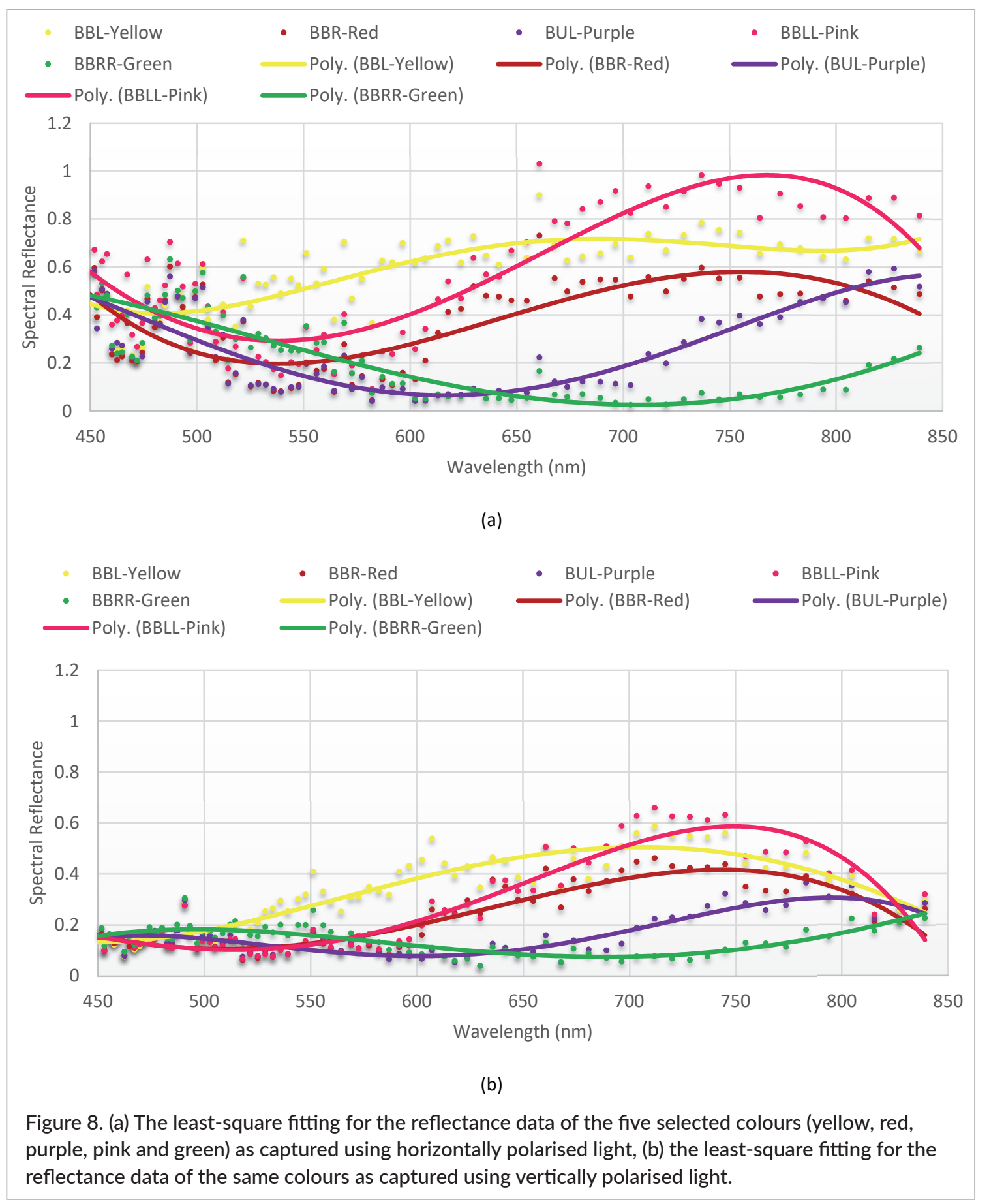

of the CCP target. In the final analysis, it is useful to utilise a spectropolarimeter for visualising intricate, man-made and natural objects to preserve all contained information in the targeted FOV.

\section{Conclusions}

The purpose of developing the spectropolarimeter, in this study, is to provide two simultaneous cross-polarised spectral images of the object of interest. The provided duality of spectral data provides multiple advantages: first, it achieves high optical throughput, as proved earlier; ${ }^{50}$ second, the new configuration creates a cost-effective imaging spectropolarimeter in comparison to the commercial VNIR ones; third, and finally, it allows real-time monitoring of an object using two cross-polarised views across the entire operating spectral range. For instance, the developed setup might be used for monitoring the fluorescence emission of a medical/non-medical tissue sample, expressly if it has a polarisation preference. 


\section{References}

1. S. Kiyotoki, J. Nishikawa, T. Okamoto, K. Hamabe, M. Saito, A. Goto, Y. Fujita, Y. Hamamoto, Y. Takeuchi, S. Satori and I. Sakaida, "New method for detection of gastric cancer by hyperspectral imaging: a pilot study", J. Biomed. Opt. 18(2), 026010 (2013). https:// doi.org/10.1117/1.JBO.18.2.026010

2. R. Abdlaty, L. Doerwald, J. Hayward and Q. Fang, "Spectral assessment of radiation therapy-induced skin erythema", Proc. SPIE 11316, 1131619 (2020). https://doi.org/10.1117/12.2541515

3. E.L.P. Larsen, L.L. Randeberg, E. Olstad, O.A. Haugen, A. Aksnes and L.O. Svaasand, "Hyperspectral imaging of atherosclerotic plaques in vitro", J. Biomed. Opt 16(2), 026011 (2011). https:// doi.org/10.1117/1.3540657

4. A.S. Alenin, L. Morrison, C. Curiel and J.S. Tyo, "Hyperspectral measurement of the scattering of polarized light by skin", Proc. SPIE 8160, 816014 (2011). https://doi.org/10.1117/12.895552

5. A.F.H. Goetz and V. Srivastava, "Mineralogical mapping in the Cuprite mining district, Nevada", Proc. of the Airborne Imaging Spectrometer Data Anal. Workshop (1985). https://ntrs.nasa.gov/citations/19860002152

6. A.F.H.Goetz, G. Vane, J.E. Solomon and B.N. Rock, "Imaging spectrometry for earth remote sensing," Science 228, 11471153 (1985). https://doi. org/10.1126/science.228.4704.1147

7. H. Liang, "Advances in multispectral and hyperspectral imaging for archaeology and art conservation," Appl. Phys. A 106(2), 309-323 (2011). https://doi. org/10.1007/s00339-011-6689-1

8. R. Abdlaty, M. Gobara, I. Naiem and M. Mokhtar, "Innovative technique for analysis of wastewater contaminants using hyperspectral imaging", J. Spectral Imaging 9, a12 (2020). https://doi. org/10.1255/jsi.2020.a12

9. H. Huang, L. Liu and M.O. Ngadi, "Recent developments in hyperspectral imaging for assessment of food quality and safety", Sensors 14(4), 7248-7276 (2014). https://doi.org/10.3390/s140407248

10. J. Kuula, I. Pölönen, H.-H. Puupponen, T. Selander, T. Reinikainen, T. Kalenius and H. Saari, "Using VIS/NIR and IR spectral cameras for detecting and separating crime scene details", Proc. SPIE 8359, 83590P (2012). https://doi.org/10.1117/12.918555

11. G.J. Edelman, E. Gaston, T.G. van Leeuwen, P.J. Cullen and M.C.G. Aalders, "Hyperspectral imaging for non-contact analysis of forensic traces",
Forensic Sci. Intl. 223(1-3), 28-39 (2012). https://doi. org/10.1016/j.forsciint.2012.09.012

12. M.A. Calin, S.V. Parasca, D.A.N. Savastru and D. Manea, "Hyperspectral imaging in the medical field: present and future", Appl. Spectrosc. Rev. 49, 435447 (2014). https://doi.org/10.1080/05704928.201 3.838678

13. A.J. Brown, S.J. Hook, A.M. Baldridge, J.K. Crowley, N.T. Bridges, B.J. Thomson, G.M. Marion, C.R. de Souza Filho and J.L. Bishop, "Hydrothermal formation of Clay-Carbonate alteration assemblages in the Nili Fossae region of Mars", Earth Planet. Sci. Lett. 297, 1-21 (2010). https://doi.org/10.1016/j. epsl.2010.06.018

14. A.J. Brown, C.E. Viviano and T.A. Goudge, "Olivinecarbonate mineralogy of the Jezero Crater region", JGR: Planets 125(3), e2019JE006011 (2020). https:// doi.org/10.1029/2019JE006011

15. L. Trefan, C. Harris, S. Evans, D. Nuttall, S. Maguire and A.M. Kemp, "A comparison of four different imaging modalities - Conventional, cross polarized, infra-red and ultra-violet in the assessment of childhood bruising", J. Forensic Leg. Med. 59, 30-35 (2018). https://doi.org/10.1016/j.jflm.2018.07.015

16. S. Chaudhary, S. Ninsawat and T. Nakamura, "Nondestructive trace detection of explosives using pushbroom scanning hyperspectral imaging system", Sensors 19(1), 1-15 (2019). https://doi.org/10.3390/ s19010097

17. D.A. Glenar, J.J. Hillman, B. Saif and J. Bergstralh, "Acousto-optic imaging spectropolarimetry for remote sensing", Appl. Opt. 33(31), 7412-7424 (1994). https://doi.org/10.1364/AO.33.007412

18. J.M. Schmitt, A.H. Gandjbakhche and R.F. Bonner, "Use of polarized light to discriminate short-path photons in a multiply scattering medium", Appl. Opt. 31(30), 6535-6546 (1992). https://doi.org/10.1364/ AO.31.006535

19. E. Rizova and A. Kligman, "New photographic techniques for clinical evaluation of acne," J. Eur. Acad. Dermatol. Venereol. 15(Suppl. 3), 13-18 (2001). https://doi.org/10.1046/j.1468-3083.15.s.3.1.x

20. S.L. Jacques, J.C. Ramella-Roman and K. Lee, "Imaging skin pathology with polarized light", J. Biomed. Opt. 7(3), 329-340 (2002). https://doi. org/10.1117/1.1484498

21. N. Gupta, R. Dahmani and S. Choy, "Acousto-optic tunable filter based visible- to near-infrared spectropolarimetric imager", Opt. Eng. 41(5), 1033-1038 (2002). https://doi.org/10.1117/1.1467936 
22. A. Sviridov, V.V. Chernomordik, M. Hassan, A. Russo, A.B. Eidsath, P.D. Smith and A.H. Gandjbakhche, "Intensity profiles of linearly polarized light backscattered from skin and tissue-like phantoms", J. Biomed. Opt. 10(1), 014012 (2005). https://doi. org/10.1117/1.1854677

23. D. Kapsokalyvas, R. Cicchi, N. Bruscino, D. Alfieri, F. Prignano, D. Massi, T. Lotti and F.S. Pavone, "In-vivo imaging of psoriatic lesions with polarization multispectral dermoscopy and multiphoton microscopy", Biomed. Opt. Express 5(7), 2405-2419 (2014). https://doi.org/10.1364/BOE.5.002405

24. S. Sridhar and A. Da Silva, "Enhanced contrast and depth resolution in polarization imaging using elliptically polarized light", J. Biomed. Opt. 21(7), 071107 (2016). https://doi.org/10.1117/1.JBO.21.7.071107

25. G. Lu and B. Fei, "Medical hyperspectral imaging: a review", J. Biomed. Opt. 19(1), 10901 (2014). https:// doi.org/10.1117/1.JBO.19.1.010901

26. Q. Li, X. He, Y. Wang, H. Liu, D. Xu and F. Guo, "Review of spectral imaging technology in biomedical engineering: achievements and challenges", J. Biomed. Opt. 18(10), 100901 (2013). https://doi. org/10.1117/1.JBO.18.10.100901

27. N. Hagen and M.W. Kudenov, "Review of snapshot spectral imaging technologies", Opt. Eng. 52(9), 090901 (2013). https://doi.org/10.1117/1. OE.52.9.090901

28. P. Mishra, M.S.M. Asaari, A. Herrero-Langreo, S. Lohumi, B. Diezma and P. Scheunders, "Close range hyperspectral imaging of plants: A review", Biosyst. Eng. 164, 49-67 (2017). https://doi.org/10.1016/j. biosystemseng.2017.09.009

29. N. Gat, "Imaging spectroscopy using tunable filters: a review", Proc. SPIE 4056, 50-64 (2000). https://doi. org/10.1117/12.381686

30. T.-H. Chao, J. Yu, G. Reyes, D. Rider and L.-J. Cheng, "Acousto-optic tunable filter imaging spectrometers", IGARSS'91 Remote Sensing: Global Monitoring for Earth Management, Espoo, Finland, pp. 585-588 (1991). https://doi.org/10.1109/ IGARSS.1991.579956

31. N. Gupta, "Development of staring hyperspectral imagers", 2011 IEEE Applied Imagery Pattern Recognition Workshop (AIPR), Washington, DC, pp. 1-8 (2011). https://doi.org/10.1109/ AIPR.2011.6176379

32. T. Okamoto and I. Yamaguchi, "Simultaneous acquisition of spectral image information", Opt. Lett.
16(16), 1277-1279 (1991). https://doi.org/10.1364/ OL.16.001277

33. H. Ding, Q. Lu, H. Gao and Z. Peng, "Non-invasive prediction of hemoglobin levels by principal component and back propagation artificial neural network", Biomed. Opt. Express 5(4), 1145-1152 (2014). https:// doi.org/10.1364/BOE.5.001145

34. R. Abdlaty, S. Sahli, J. Hayward and Q. Fang, "Hyperspectral imaging: comparison of acousto-optic and liquid crystal tunable filters", Proc. SPIE 10573, 105732P (2018). https://doi. org/10.1117/12.2282532

35. R. Abdlaty and Q. Fang, "Acousto-optic tunable filter-based hyperspectral imaging system characterization," Proc. SPIE 10870, 108700Y (2019). https:// doi.org/10.1117/12.2506318

36. V.I. Batshev, A.S. Machikhin, A.B. Kozlov, S.V. Boritko, M.O. Sharikova, A.V. Karandin, V.E. Pozhar and V.A. Lomonov, "Tunable acousto-optic filter for the 450-900 and 900-1700 nm spectral range," J. Commun. Technol. Electron. 65(7), 800-805 (2020). https://doi.org/10.1134/S1064226920070025

37. W. Wang, C. Li, E.W. Tollner, G.C. Rains and R.D. Gitaitis, "A liquid crystal tunable filter based shortwave infrared spectral imaging system: Design and Integration", Comput. Electron. Agric. 80, 135-144 (2012). https://doi.org/10.1016/j.compag.2011.09.003

38. S.C. Gebhart, R.C. Thompson and A. MahadevanJansen, "Liquid-crystal tunable filter spectral imaging for brain tumor demarcation", Appl. Opt. 46(10), 1896 (2007). https://doi.org/10.1364/ A0.46.001896

39. O. Aharon, I. Abdulhalim, O. Arnon, L. Rosenberg, V. Dyomin and E. Silberstein, "Differential optical spectropolarimetric imaging system assisted by liquid crystal devices for skin imaging", J. Biomed. Opt. 16(8), 086008 (2011). https://doi. org/10.1117/1.3609003

40. R.W. Slawson, Z. Ninkov and E.P. Horch, "Hyperspectral imaging: wide-area spectrophotometry using a liquid-crystal tunable filter hyperspectral imaging", Publ. Astron. Soc. Pacific 111(759), 621-626 (2014). https://doi.org/10.1086/316360

41. D.N. Stratis, K.L. Eland, J.C. Carter, S.T. Tomlinson and S.M. Angel, "Comparison of acousto-optic and liquid crystal tunable filters for laser-induced breakdown spectroscopy," Appl. Spectrosc. 55(8), 999-1004 (2001). https://doi. org/10.1366/0003702011953144 
42. H.R. Morris, C.C. Hoyt and P.J. Treado, "Imaging spectrometers for fluorescence and raman microscopy: acousto-optics and liquid crystal tunable filters", Appl. Spectrosc. 48(7), 857-866 (1994). https:// doi.org/10.1366/0003702944029820

43. W.M. Hayden Smith and K.M. Smith, "A polarimetric spectral imager using acousto-optic tunable filters", Exp. Astron. 1(5), 329-343 (1990). https://doi. org/10.1007/BF00454329

44. M.A. Sturgeon, L.-J. Cheng, P.H. Durkee, M.K. Hamilton, J.F. Huth, J.C. Mahoney, R.C. Olsen and G.F. Reyes, "Spectral and polarimetric analysis of hyperspectral data collected by an acousto-optic tunable filter system", Proc. SPIE 2231, 167-176 (1994). https://doi.org/10.1117/12.179777

45. A.J. Brown, "Equivalence relations and symmetries for laboratory, LIDAR, and planetary Müeller matrix scattering geometries", J. Opt. Soc. Amer. A 31(12), 2789-2794 (2014). https://doi.org/10.1364/ JOSAA.31.002789

46. F. Li, Y. Xu and Y. Ma, "Design of hyper-spectral and full-polarization imager based on AOTF and
LCVR", Proc. SPIE 9298, 92980 (2014). https://doi. org/10.1117/12.2073012

47. G. Georgiev, D.A. Glenar and J.J. Hillman, "Spectral characterization of acousto-optic filters used in imaging spectroscopy", Appl. Opt. 41(1), 209-217 (2002). https://doi.org/10.1364/A0.41.000209

48. J.E. Garcia, M.B. Girard, M. Kasumovic, P. Petersen, P.A. Wilksch and A.G. Dyer, "Differentiating biological colours with few and many sensors: spectral reconstruction with RGB and hyperspectral cameras", PLoS One 10(5), e0125817 (2015). https://doi. org/10.1371/journal.pone.0125817

49. C.A. Schneider, W.S. Rasband and K.W. Eliceiri, "NIH Image to Image J: 25 years of image analysis", Nat. Methods 9, 671-675 (2012). https://doi.org/10.1038/ nmeth.2089

50. R. Abdlaty, J. Orepoulos, P. Sinclair, R. Berman and Q. Fang, "High throughput AOTF hyperspectral imager for randomly polarized light", Photonics 5(1), 3 (2018). https://doi.org/10.3390/photonics5010003 